An approximate single fluid 3-dimensional magnetohydrodynamic equilibrium model with toroidal flow

This content has been downloaded from IOPscience. Please scroll down to see the full text.

2014 Plasma Phys. Control. Fusion 56094004

(http://iopscience.iop.org/0741-3335/56/9/094004)

View the table of contents for this issue, or go to the journal homepage for more

Download details:

IP Address: 128.178.125.173

This content was downloaded on 14/08/2014 at $11: 42$

Please note that terms and conditions apply. 


\title{
An approximate single fluid 3-dimensional magnetohydrodynamic equilibrium model with toroidal flow
}

\author{
W A Cooper ${ }^{1}$, S P Hirshman ${ }^{2}$, I T Chapman ${ }^{3}$, D Brunetti ${ }^{1}$, J M Faustin ${ }^{1}$, \\ J P Graves ${ }^{1}$, D Pfefferlé ${ }^{1}$, M Raghunathan ${ }^{1}$, O Sauter ${ }^{1}$, T M Tran ${ }^{1}$ and \\ N Aiba ${ }^{4}$ \\ ${ }^{1}$ Ecole Polytechnique Fédérale de Lausanne (EPFL), Centre de Recherches en Physique des Plasmas, \\ Association Euratom-Confédération Suisse, CH1015 Lausanne, Switzerland \\ ${ }^{2}$ Fusion and Nuclear Materials Sciences Division, Oak Ridge National Laboratory, Oak Ridge, \\ TN 37831, USA \\ ${ }^{3}$ Euratom/CCFE Fusion Association, Culham Science Centre, Abingdon, Oxon OX14 3DB, UK \\ ${ }^{4}$ Japan Atomic Energy Authority, Rokkasho, Aomori 039-3212, Japan \\ E-mail: wilfred.cooper@epfl.ch
}

Received 5 December 2013, revised 11 February 2014

Accepted for publication 31 March 2014

Published 13 August 2014

\begin{abstract}
An approximate model for a single fluid three-dimensional (3D) magnetohydrodynamic (MHD) equilibrium with pure isothermal toroidal flow with imposed nested magnetic flux surfaces is proposed. It recovers the rigorous toroidal rotation equilibrium description in the axisymmetric limit. The approximation is valid under conditions of nearly rigid or vanishing toroidal rotation in regions with significant 3D deformation of the equilibrium flux surfaces. Bifurcated helical core equilibrium simulations of long-lived modes in the MAST device demonstrate that the magnetic structure is only weakly affected by the flow but that the 3D pressure distortion is important. The pressure is displaced away from the major axis and therefore is not as noticeably helically deformed as the toroidal magnetic flux under the subsonic flow conditions measured in the experiment. The model invoked fails to predict any significant screening by toroidal plasma rotation of resonant magnetic perturbations in MAST free boundary computations.
\end{abstract}

Keywords: toroidal rotation, magnetohydrodynamic equilibrium, three-dimensional

(Some figures may appear in colour only in the online journal)

\section{Introduction}

Long-lived modes (LLMs) are observed in MAST that appear to correspond to ideal magnetohydrodynamic (MHD) structures similar to saturated internal kink modes as shown by the soft $x$-ray experimental fluctuations in figure $1[1,2]$. The toroidal rotation in the core decreases from $\sim 150 \mathrm{~km} \mathrm{~s}^{-1}$ at the mode onset to about $\sim 100 \mathrm{~km} \mathrm{~s}^{-1}$ when the LLM is well established after $\sim 30 \mathrm{~ms}$ and the toroidal velocity profile increases almost linearly with major radius $R$. This indicates that the toroidal flow, at least in the central region of the plasma, becomes almost rigid within the three-dimensional (3D) domain in which the LLM exists. Outside the core, the rotation velocity is more clearly sheared. Similar stationary saturated structures are observed in NSTX [3]. Application of external resonant magnetic perturbations (RMPs) tends to suppress the toroidal plasma rotational by almost an order of magnitude or larger depending on the toroidal mode number of the applied field [4]. The measured toroidal rotation frequency $\Omega$ is plotted at the midplane as a function of $R$ for external perturbations with toroidal mode number $N=3,4$ and 6 from the magnetic axis (at $R \sim 0.9 \mathrm{~m}$ ) to the edge of the plasma (at $R \sim 0.4 \mathrm{~m}$ ) in figure 2. The suppression of rotation is largest for $N=3$, though there appears to be a reversal of the flow near mid-radius. The main point of these observations is that the toroidal rotation is either suppressed or survives but becomes rigid when there are 3D deformations in the plasma. The detection of LLMs and snakes is possible 


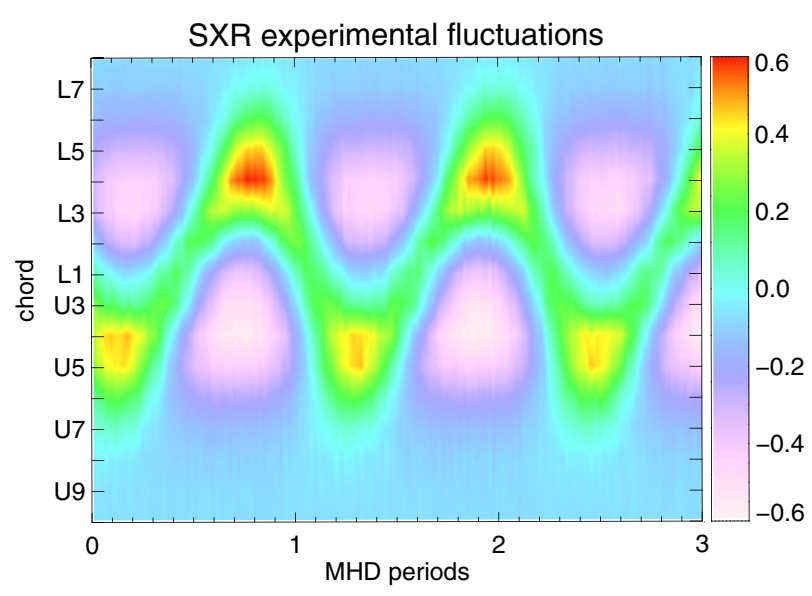

Figure 1. The soft $x$-ray experimental fluctuations in MAST shows a saturated $n=1$ perturbation rotating in the core.

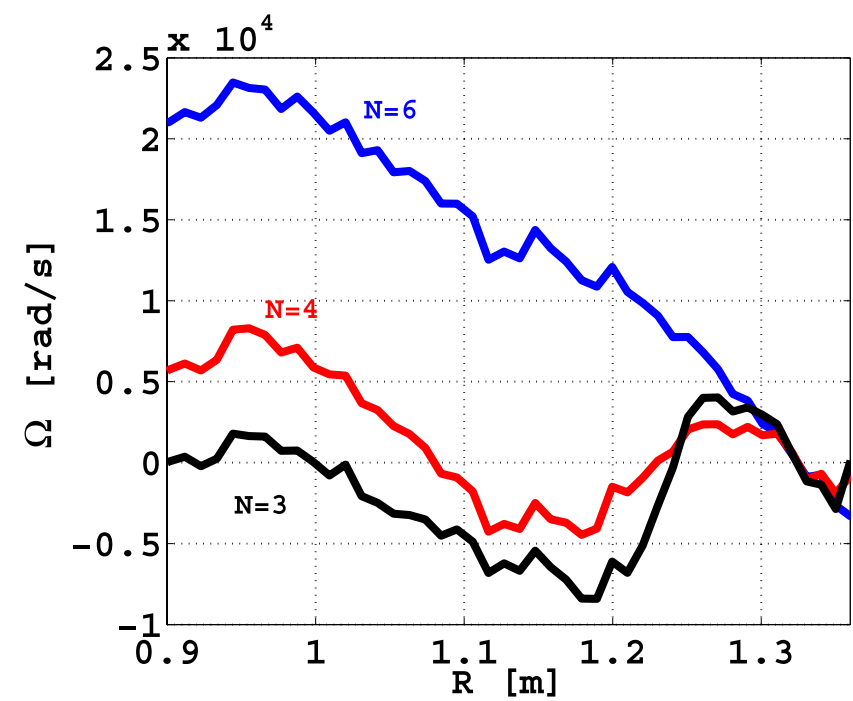

Figure 2. The toroidal angular rotation frequencies as a function of $R$ (the distance from the major axis) for externally applied RMP mode numbers $N=3$ (shot \#27654, lower black curve), $N=4$ (shot \#27846, middle red curve) and $N=6$ (shot \#27204, upper blue curve) at $t=0.29 \mathrm{~s}$.

in experiments because the mode structure rotates toroidally about the observation chords of the diagnostics.

The equilibrium state with toroidal flow in a tokamak at finite $\langle\beta\rangle$ was first analysed in [5]. Recently, a combination of toroidal flow and pressure anisotropy in an axisymmetric plasma has been formulated, implemented in the EFIT code and applied to MAST [6]. A different formulation of the problem is also worth noting [7]. Applications including poloidal flow in an axisymmetric tokamak have been reported [8] using the code FLOW [9] based on equations derived in [10]. However, the results obtained are clouded in some controversy because singularities arise at relatively modest poloidal Mach number in the single fluid model considered that are not manifest in more complete multifluid/kinetic descriptions [11]. This model is also the basis for the CLIO code [12]. The M3D hybrid MHD-kinetic code has been previously applied to NSTX axisymmetric equilibria and quite accurately recovers features of the density profile that is displaced radially outward when the toroidal rotation is strong [13]. Helical $m=1, n=1$ impurityinduced snakes that rotate at the same rate as the toroidal flow have been reported in Alcator C-Mod [14] and simulations with an extended version of the M3D code seem to agree with the experimental observations [15]. We interpret snakes and LLM modes as manifestations of the same physical phenomenon, basically saturated $m=1, n=1$ internal kink structures. The linear and nonlinear stability of stationary isothermal axisymmetric equilibrium states in NSTX have also been investigated with the M3D code that yield saturated core structures very similar to the helical core equilibria we compute [16].

The principal issues we wish to at least partially address in this paper focus on how the toroidal rotation affects the helical core bifurcation associated with LLM simulations and the screening effect of toroidal rotation in RMP simulations. An approximate model for stationary 3D MHD equilibria with pure toroidal flow constrained to have nested magnetic flux surfaces is formulated in section 2. Fixed boundary bifurcated MHD equilibria with a 3D helical core that models LLM structures in MAST with toroidal rotation is presented in section 3. Free boundary 3D MHD equilibrium computations in MAST with externally applied $N=3$ RMP deformations are described in section 4 . Numerical issues are addressed in section 5. A summary, conclusions and discussions appear in section 6 .

\section{Approximate 3D equilibrium with toroidal flow}

The MHD force balance relation under static conditions is

$$
\begin{aligned}
\mu_{0} \boldsymbol{F} & =-\mu_{0} \nabla p+\mu_{0} \boldsymbol{j} \times \boldsymbol{B} \\
& =-\mu_{0} \nabla p+(\boldsymbol{\nabla} \times \boldsymbol{B}) \times \boldsymbol{B}=0 .
\end{aligned}
$$

This implies that the parallel force balance projection yields

$$
\boldsymbol{B} \cdot \nabla p=0 .
$$

In axisymmetry, this further implies that $p=p(s)$, where the radial variable: $0 \leqslant s \leqslant 1$ is chosen proportional to the magnetic flux $2 \pi \Phi$. In a $3 D$ configuration with nested magnetic flux surfaces, we impose $p=p(s)$ and we can define the energy functional [17-19]

$$
\mu_{0} W=\iiint \mathrm{d}^{3} x\left(\frac{B^{2}}{2}+\mu_{0} \frac{p(s)}{\Gamma-1}\right) .
$$

The first variation of the energy functional yields the MHD equilibrium state, where $\Gamma$ is the adiabatic index and $B$ is the magnetic field strength. The MHD force balance in the presence of plasma rotation is written as

$$
\mu_{0} \boldsymbol{F}=-\mu_{0} \nabla p-\mu_{0} \rho_{M}(\boldsymbol{V} \cdot \nabla) \boldsymbol{V}+(\nabla \times \boldsymbol{B}) \times \boldsymbol{B}=0 .
$$

For pure toroidal flow, the combination of Faraday's Law and Ohm's Law under ideal conditions is written as $\boldsymbol{\nabla} \times(\boldsymbol{V} \times \boldsymbol{B})=$ 0 and the $\nabla \phi$ projection becomes $\nabla \cdot\left(B V^{\phi}-V B^{\phi}\right)=0$. Applying the continuity equation $\nabla \cdot\left(\rho_{M} \boldsymbol{V}\right)=0$ implies that

$$
\boldsymbol{B} \cdot \nabla V^{\phi}=\rho_{M} V^{\phi} \frac{\partial}{\partial \phi}\left(\frac{B^{\phi}}{\rho_{M}}\right) .
$$


The right hand side vanishes when $\phi$ is an ignorable coordinate (like in axisymmetry or helical symmetry) and consequently the contravariant component of the velocity field in the toroidal direction $V^{\phi} \equiv \boldsymbol{V} \cdot \nabla \phi$ is a flux surface quantity, namely,

$$
\boldsymbol{V} \cdot \nabla \phi=\Omega(s),
$$

where $\Omega$ is the toroidal angular frequency. As a result, it is easy to derive that

$$
(\boldsymbol{V} \cdot \nabla) \boldsymbol{V}=\frac{1}{2} \Omega^{2}(s) \nabla R^{2} .
$$

Consider writing $p=\left(N_{i}+N_{e}\right) T=2 N T$ and $\rho_{M}=M_{i} N_{i}=$ $M_{i} N$, then $\rho_{M}=M_{i} p /(2 T)$. Large heat flow along the magnetic field lines equilibrates the temperature on a flux surface. The condition $(\boldsymbol{B} \cdot \boldsymbol{\nabla}) T=0$ yields in axisymmetry $T=T(s)$ which corresponds to isothermal conditions [20]. With this in mind, the parallel force balance becomes

$$
\boldsymbol{B} \cdot \nabla \ell n p=(\boldsymbol{B} \cdot \nabla)\left[\frac{M_{i}}{4 T(s)} \Omega^{2}(s) R^{2}\right],
$$

which we integrate to obtain

$$
p(s, R)=P_{0}(s) \exp \left[\frac{M_{i}}{4 T(s)} \Omega^{2}(s) R^{2}\right] .
$$

In axisymmetry, the energy functional

$$
\mu_{0} W=\iiint \mathrm{d}^{3} x\left[\frac{B^{2}}{2}+\frac{\mu_{0} p(s, R)}{\Gamma-1}\right]
$$

can be defined the first variation of which yields the equilibrium state with isothermal toroidal flow [21].

This procedure cannot be easily generalized to threedimensions. As a first step, we postulate that the energy functional of equation (10) is also applicable in 3D. We explore conditions for which this energy functional can approximate a 3D stationary MHD equilibrium state under isothermal conditions, namely, $T=T(s), p(s, R)=2 N(s, R) T(s)$. We assume pure toroidal flow in cylindrical coordinates, so

$$
\boldsymbol{V}=R V^{\phi} \nabla Z \times \nabla R=R^{2} V^{\phi} \nabla \phi,
$$

where we impose that $V^{\phi} \equiv \boldsymbol{V} \cdot \nabla \phi=\Omega(s)$ remains a flux surface quantity. We thus do not invoke the combination of Faraday's Law, Ohm's Law and the continuity equation to derive this relation (which results when there is an ignorable coordinate). The MHD force balance relation given by equation (4) becomes in $3 \mathrm{D}$

$$
\begin{aligned}
\mu_{0} \boldsymbol{F}= & -\mu_{0} \nabla p-\frac{1}{2} \mu_{0} \rho_{M} \Omega^{2}(s) \nabla R^{2}+(\nabla \times \boldsymbol{B}) \times \boldsymbol{B} \\
& -\mu_{0} \rho_{M} R^{2} \Omega^{\prime}(s)(\boldsymbol{V} \cdot \nabla s) \nabla \phi=0 .
\end{aligned}
$$

The first three terms are the same as those that appear in axisymmetric conditions. The last term is inherent to the $3 \mathrm{D}$ description we consider and prevents that the energy functional described in equation (10) that we have postulated would recover an equilibrium state consistent with MHD force balance. Observations of the LLMs in MAST shows that the toroidal rotation is flattened in the core region measured from the charge exchange recombination spectroscopy (CERS) diagnostic, from the magnetic axis at $R \sim 0.91 \mathrm{~m}$ to the extent of the LLM at $R \sim 1.15 \mathrm{~m}$ [1]. The toroidal rotation speed drops from $\sim 150$ to $\sim 100 \mathrm{~km} \mathrm{~s}^{-1}$ and its profile becomes approximately linear with $R$. This indicates that the toroidal rotation survives the 3D distortions induced by the LLM, but the flow becomes roughly rigid within the LLM structure. Beyond $R=1.15 \mathrm{~m}$, the toroidal flow decreases to the edge of the plasma. The details described can be seen in figure 3 of [1]. The application of externally applied RMP also has a big impact on toroidal plasma rotation in MAST. The rotation is suppressed, or decreased to the level at the pedestal depending on the magnitude of the RMP coil currents and the toroidal mode number that is applied as shown in figure 2. The main implications of these experimental observations in MAST is that spontaneous internal or externally applied 3D deformations cause the toroidal flow to become rigid or to vanish. This suggests that in regions of 3D plasma deformation $\Omega(s) \sim$ constant, but shear in the flow $\Omega^{\prime}(s) \neq 0$ can be sustained in domains that remain essentially axisymmetric (like the outer plasma regions with LLM or well inside the pedestal with RMP).

The experimental observations we have described motivate the application of some further approximations. A re-examination of equation (12) suggests that in regions with significant $3 \mathrm{D}$ distortion $\Omega^{\prime}(s) \simeq 0$, while in domains that remain roughly axisymmetric, $\boldsymbol{V} \cdot \nabla s \simeq 0$. Invoking these approximations, the last term of equation (12) is negligible compared with the other terms. As a result, the first variation of the energy functional given in equation (10) is consistent with a $3 \mathrm{D}$ equilibrium description of a plasma with toroidal rotation. This formulation of the 3D MHD equilibrium problem with toroidal plasma rotation and nested magnetic fluix surfaces has been implemented as an extension of the VMEC equilibrium code [17-19]. It is worth noting that it is convenient to define the function $U$ as

$$
U(s)=\frac{M_{i}}{4 T(s)} \Omega^{2}(s),
$$

and then normalize the toroidal rotation frequency $\Omega(s)$ / $\Omega(0) \Longrightarrow \Omega(s)$ and the temperature $T(s) / T(0) \Longrightarrow T(s)$. Then we can write

$$
U(s)=\frac{\mathcal{M}_{0}^{2}}{2 R_{0}^{2}} \frac{\Omega^{2}(s)}{T(s)},
$$

where $\mathcal{M}_{0}$ is the Mach number at the magnetic axis and $R_{0}$ is the toroidally averaged value of $R$ on axis.

\section{Fixed boundary MAST LLM simulations}

Bifurcated helical core equilibrium states are obtained with toroidal current profiles that are slightly hollow and these generate $q$-profiles with weakly reversed central magnetic shear and $q_{\text {min }}$ near unity around mid-radius [22-25]. For cases with toroidal rotation, we adopt the profiles which are shown in figure 3. 


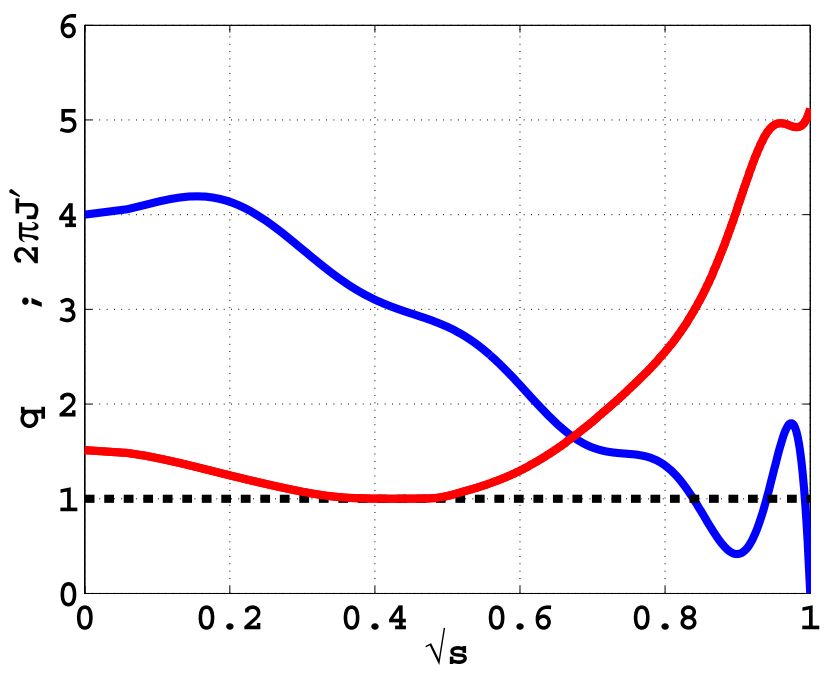

Figure 3. The toroidal current profile $2 \pi J^{\prime}$ and the corresponding $q$-profile as a function of $\sqrt{s}(s \propto$ enclosed toroidal magnetic flux, so $\sqrt{s}$ is a measure of the normalized plasma radius). The toroidal current has an edge bootstrap component which induces a wiggle in the $q$-profile.

The normalized toroidal rotation frequency and temperature profiles as a function of $\sqrt{s}$ ( $s$ is roughly proportional to the enclosed volume) are plotted in figure 4 . We have deliberately chosen the toroidal rotation frequency $\Omega(s)$ and $T(s)$ profiles to be flat in the core of the plasma consistent with the experimental MAST observations. The $\Omega(s)$ profile is expressed as $\left(1-s^{5}\right)^{4}$ and the $T(s)$ profile is prescribed as $\left(1-s^{4}\right)^{5}$. We have succeeded to obtain a bifurcated equilibrium solution with finite toroidal rotation and Mach number $\mathcal{M}_{0}=0.3$. The toroidal magnetic flux contours at four different cross sections with angles $\phi=0, \pi / 3,2 \pi / 3$ and $\pi$ that encompasses half a toroidal transit are displayed in figure 5. It shows a clear helical structure in the inner half of the plasma surrounded by an axisymmetric mantle in the outer half. The volume averaged $\langle\beta\rangle$ is $5.09 \%$ and the toroidal current is $603 \mathrm{kA}$. The pressure contours also display a 3D distortion in the core, but due to the $\exp \left(U R^{2}\right)$ effect, they are displaced radially outward from the major axis of MAST. The peak of the pressure has a much smaller helical deformation than that of the toroidal magnetic flux. This is shown on the same toroidal cross-sections in figure 6. It is also clear that the mass density $\rho_{M}$ is distributed in a similar manner. The magnitude of the helical core deformation is most sensitively dependent on the proximity of $q_{\min }$ to unity and to the radial location of $q_{\text {min }} \sim 1$, even if the centrifugal force acts to diminish the peak pressure distortion.

In order to better appreciate the effect of rotation on the pressure distribution, we compute a bifurcated helical core equilibrium state without flow $\mathcal{M}_{0}=0$. The $\mathcal{M}_{0}=0$ pressure contours, shown in figure 7 , have an allure that is closer to that of the magnetic flux contours at $\mathcal{M}_{0}=0.3$. These pressure contours are less dense in the core of the plasma than those of the toroidal magnetic flux because the pressure profile is quite flat in the centre of the plasma. For the sake of completeness, we present the contours of the rotational energy $0.5 \rho_{M} \Omega^{2} R^{2}$ on the same four cross-sections in figure 8 . These appear essentially axisymmetric and correspond to

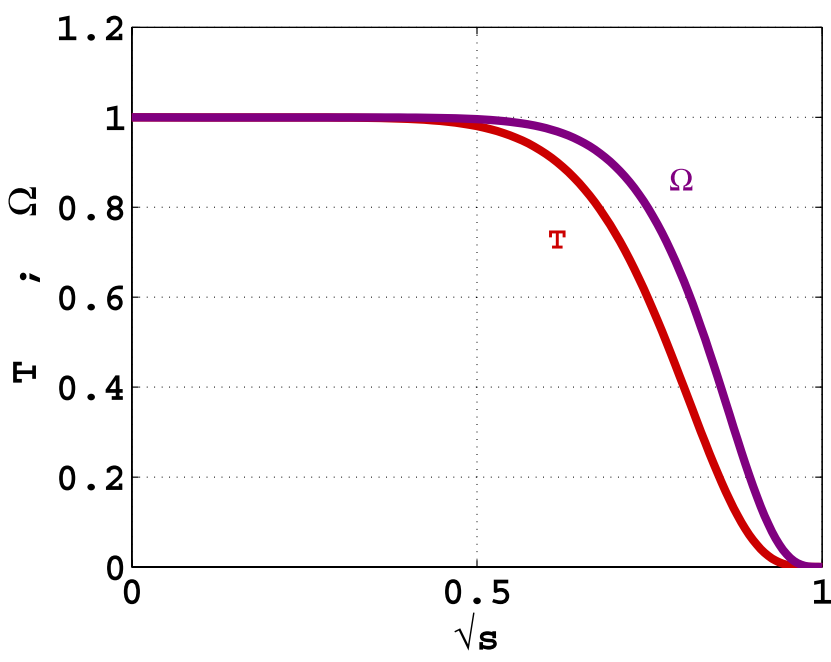

Figure 4. The standard profiles for the toroidal rotation frequency $\Omega(s)=\left(1-s^{5}\right)^{4}$ and the temperature $T(s)=\left(1-s^{4}\right)^{5}$ as a function of $\sqrt{s}$.

$U(s) R^{2} p(s, R)$. The centrifugal force pushes the rotational energy strongly away from the major axis. However, if we define $\left\langle\beta_{R}\right\rangle=\mu_{0}\left\langle\rho_{M} \Omega^{2} R^{2}\right\rangle /\left\langle B^{2}\right\rangle$, this is actually very small at $0.35 \%$ compared with the $\langle\beta\rangle \simeq 5.1 \%$ due to the pressure.

The equilibrium model that we apply can depend on gradients of the function $U(s)$, but not directly on the gradients of $\Omega$ and $T$. As we have intentionally chosen $\left|\Omega^{\prime}(s) / \Omega\right| \ll 1$ in the core, we can alter the gradients of $U$ by varying the $T$ profile. We have undertaken simulations with a flat $T$-profile, with a parabolic $T$-profile and with a hollow $T$-profile which is displayed in figure 9. The resulting $U$ profiles for the calculations appear in figure 10. The standard $U$-profile is flat in the core. The case with parabolic $T$-profile yields a hollow core $U$-profile, so $U^{\prime}>0$ in the centre of the plasma. The hollow $T$-profile produces a monotonically decreasing $U$-profile everywhere. However, we detect no significant differences in the pressure distribution in the core of the plasma due to positive, vanishing or negative gradients in the $U(s)$ function.

\section{Free boundary MAST RMP simulations}

We investigate free boundary MAST equilibria with externally applied $N=3$ up-down symmetric RMP coil currents. The volume averaged $\langle\beta\rangle \simeq 6.1 \%$ and the toroidal plasma current is $780 \mathrm{kA}$. The pressure and toroidal current profiles are quite similar to those of the fixed boundary calculations we reported in the previous section. The temperature profile is chosen to be $T(s)=0.65(1-s)\left(1-s^{2}\right)+0.35\left(1-s^{20}\right)$ which yields a monotonically decreasing curve with an edge temperature pedestal. The toroidal current profile has a corresponding edge bootstrap component. We compare in figure 12 the nonaxisymmetric contributions to $B$ of cases with $\mathcal{M}_{0}=0$, a broad $\Omega(s)=\left(1-s^{6}\right)^{3}$ profile with $\mathcal{M}_{0}=0.3$ and a more peaked $\Omega(s)=\left(1-s^{3}\right)^{6}$ profile with $\mathcal{M}_{0}=0.3$. We consider a set of $\Omega(s)$ profiles with different values of $\Omega$ at the edge of the plasma. The numerical results show that there is no significant effects of varying the edge rotation on the final 


$$
\begin{aligned}
& 0000 \\
& 0000 \\
& 0000
\end{aligned}
$$



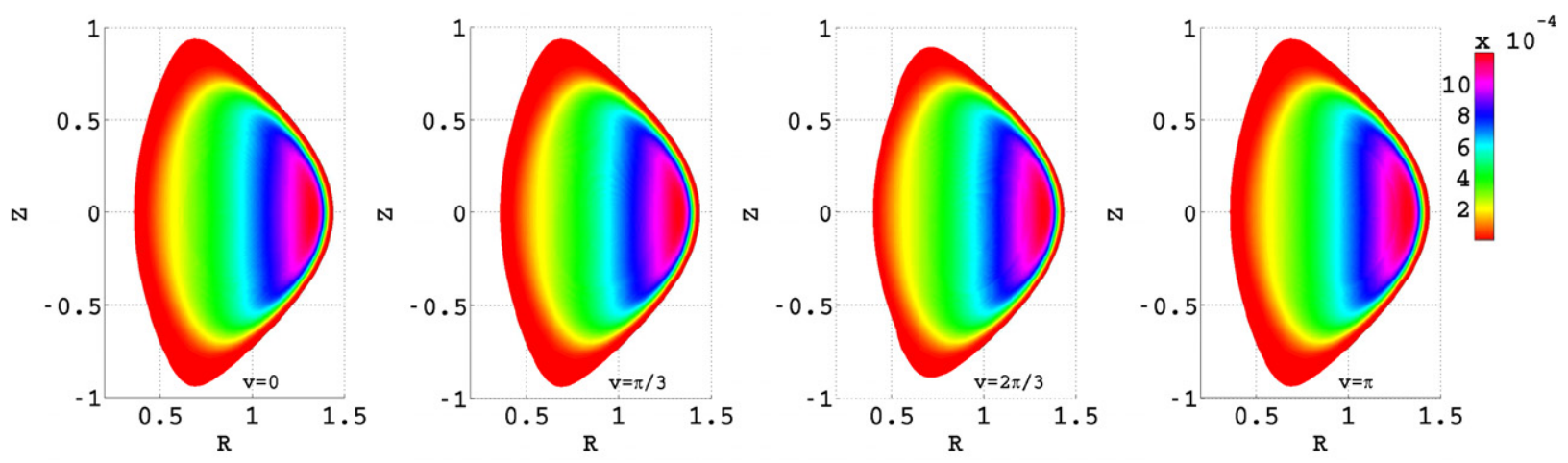

Figure 8. The rotational energy contours for the helical branch solution computed with the extended VMEC model with rotation we have implemented for a fixed boundary MAST configuration at $\langle\beta\rangle=5.09 \%$, toroidal current of $603 \mathrm{kA}$ and $\mathcal{M}_{0}=0.3$ at fixed cross-sections with toroidal angle $\phi=0$ (far left), $\phi=\pi / 3$ (middle left), $\phi=2 \pi / 3$ (middle right) and $\phi=\pi$ (far right). These cross-sections encompass half of the torus.

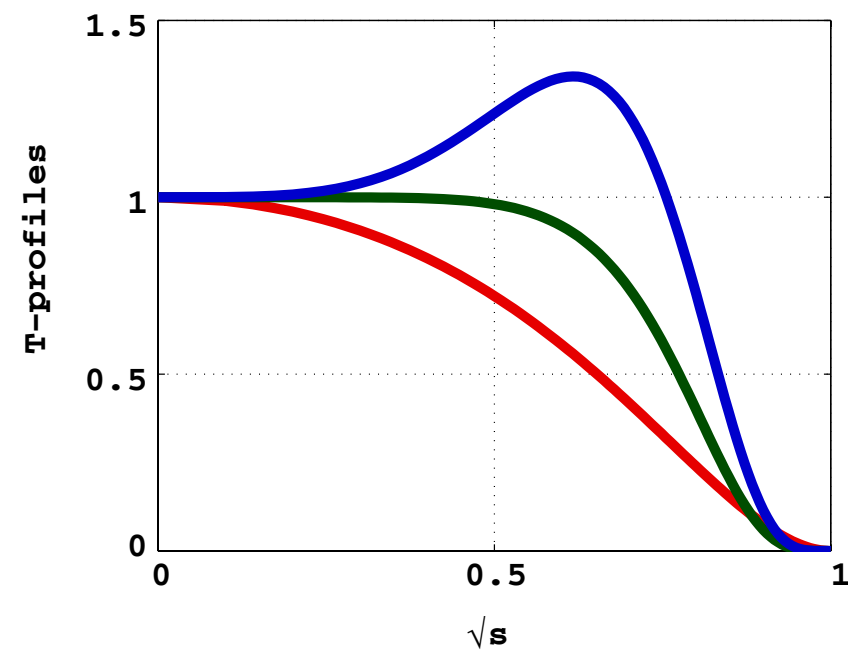

Figure 9. The temperature profiles as a function of $\sqrt{s}$ for the standard flat core case (middle green curve), for the parabolic case (lower red curve) and for the hollow case (top blue curve).

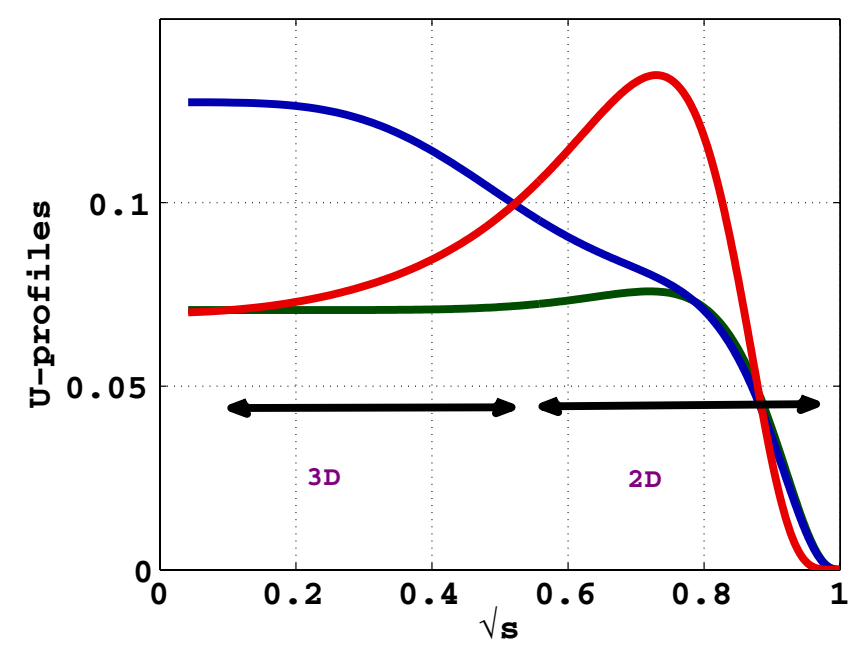

Figure 10. The $U$-function profiles as a function of $\sqrt{s}$ for fixed $\Omega(s)=\left(1-s^{5}\right)^{4}$ and parabolic $T$ (upper hollow red curve), flat core $T$ (lower green curve) and hollow $T$ (monotonically decreasing blue curve).

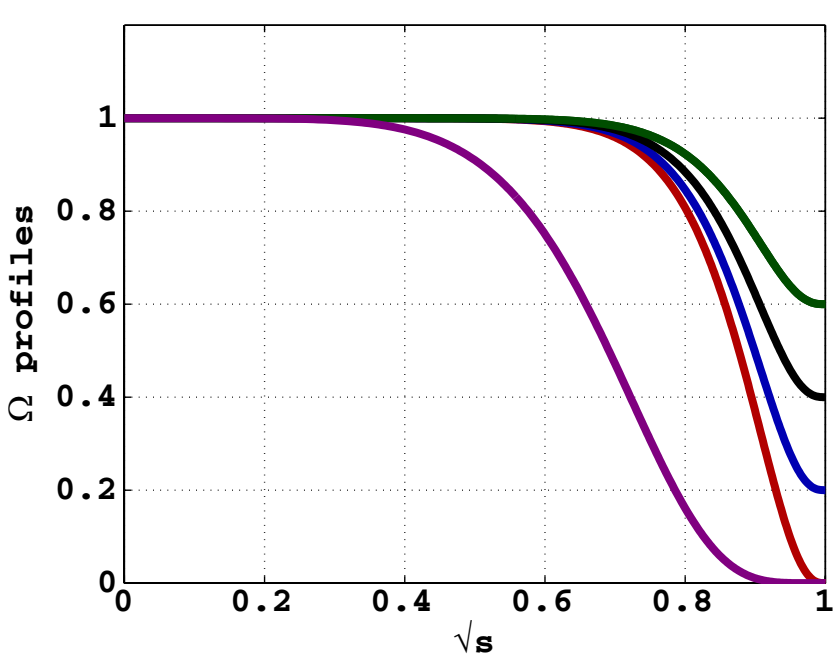

Figure 11. The rotation frequency profiles as a function of $\sqrt{s}$. The standard profile with vanishing edge $\Omega$ is given by $\Omega(s)=\left(1-s^{6}\right)^{3}$ (red curve; fourth from top). The green (top), black (second from top) and blue (third from top) curves are based on this profile, but with constant edge $\Omega$ values. The more peaked profile of $\Omega$ (in purple) is proportional to $\Omega(s)=\left(1-s^{3}\right)^{6}$.

force $\left\langle F_{R}\right\rangle$ for the fixed boundary MAST computation with the hollow temperature profile that was presented in figure 9 is displayed in figure 13. As a diagnostic of the quality of the equilibrium state achieved, the radial force balance relation $\left\langle\frac{F_{s}}{\Phi^{\prime}(s)}\right\rangle=-\left\langle\left.\frac{1}{\Phi^{\prime}(s)} \frac{\partial p}{\partial s}\right|_{R}\right\rangle-\frac{\partial}{\partial s}\left\langle\frac{\sigma B_{\phi}}{\sqrt{g}}\right\rangle-\iota(s) \frac{\partial}{\partial s}\left\langle\frac{\sigma B_{\theta}}{\sqrt{g}}\right\rangle$,

is evaluated where $\iota(s)=1 / q(s), \theta$ is the poloidal angle, $B_{\theta}$ and $B_{\phi}$ are the poloidal and toroidal field projections in the covariant representation, respectively, and the notation $\langle A\rangle$ denotes a flux surface average of $A$. After the application of the preconditioner, the convergence obtained is almost quartic with the radial mesh grid employed as shown in figure 14 .

We have detected neither shock formations, nor singularities nor discontinuities from the appearance of hyperbolic layers in the background elliptic character of 


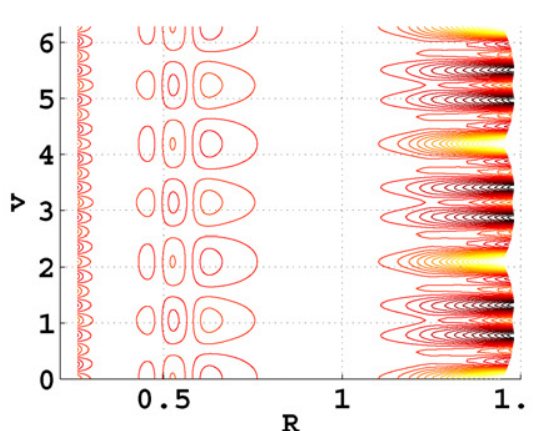

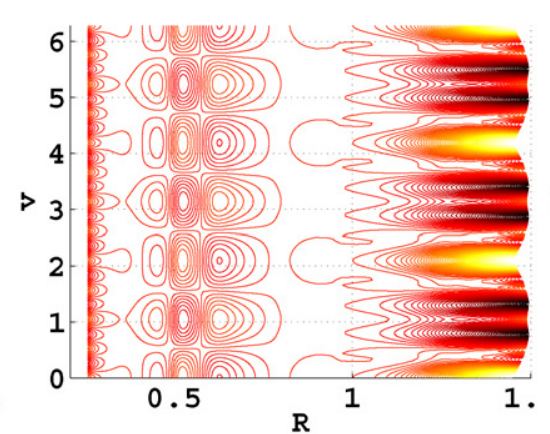

$\mathbf{R}$

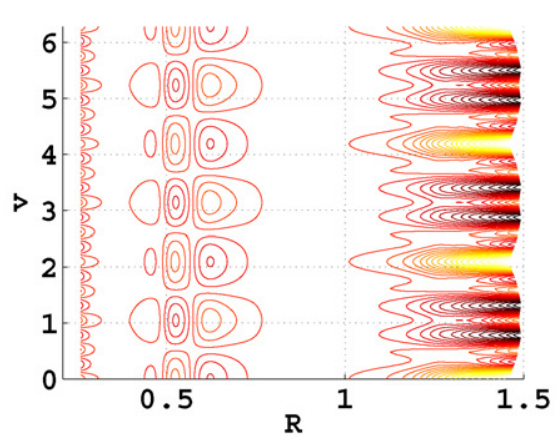

$\mathbf{R}$

Figure 12. Contours of constant sum of nonaxisymmetric contributions to $B$ in the toroidal angle $\phi(=v)$ versus $R$ at the midplane for a static plasma $\mathcal{M}_{0}=0$ (left), for a broad $\Omega$ profile at $\mathcal{M}_{0}=0.3$ (middle) and for a peaked $\Omega$ profile with $\mathcal{M}_{0}=0.351$. The current in the $n=3 \mathrm{RMP}$ coils is $5.6 \mathrm{kA}$.

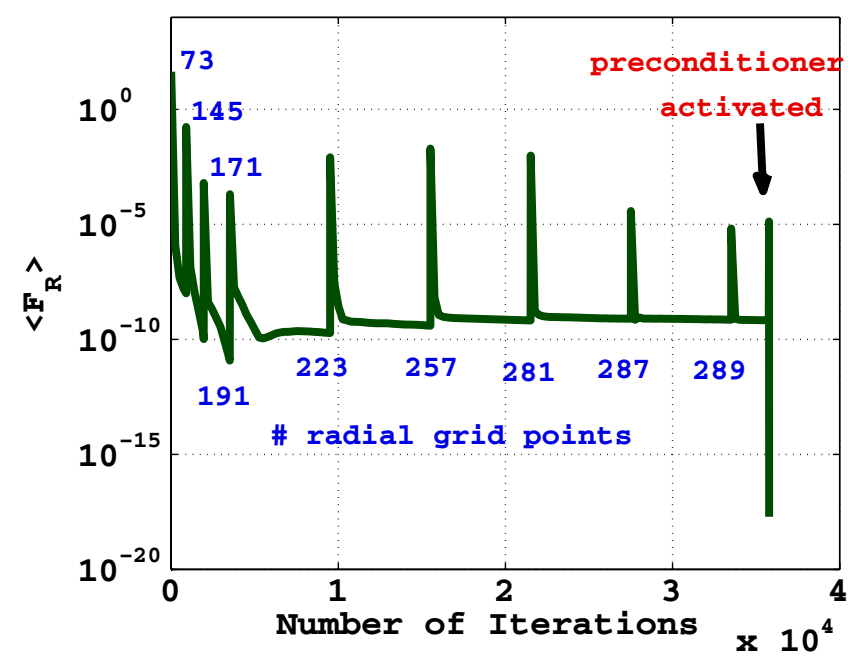

Figure 13. The averaged horizontal force $\left\langle F_{R}\right\rangle$ as a function of the number of iterations. The spikes align with changes in the number of radial grid points in the computation which are varied from 73 to 289 . The final spike aligns with the call to the preconditioner that diminishes $\left\langle F_{R}\right\rangle$ to around $10^{-18}$.

the equilibrium equations, although they could have been expected. These correspond to unphysical artifacts of single fluid MHD theory [11].

\section{Summary, conclusions and discussion}

We have derived an approximate single fluid model that describes a 3D MHD equilibrium state with isothermal toroidal plasma rotation in which nested magnetic flux surfaces are imposed. This model has been implemented as an extension of the 3D VMEC equilibrium solver [19] with finite toroidal flow. It is valid under conditions for which $\Omega^{\prime} \simeq 0$ (the radial gradient of the toroidal rotation frequency $\Omega$ ) in regions of significant 3D deformation, whether these are externally driven through RMP or spontaneously appear in the internal core of the plasma like snake-like structures or LLM. The model recovers exactly the rigorous axisymmetric isothermal toroidal flow limit. In this model, the rotation depends specifically on the flux surface function $U(s) \propto \Omega^{2}(s) / T(s)$. Consequently, shear in the function $U$ rather than in the toroidal rotation

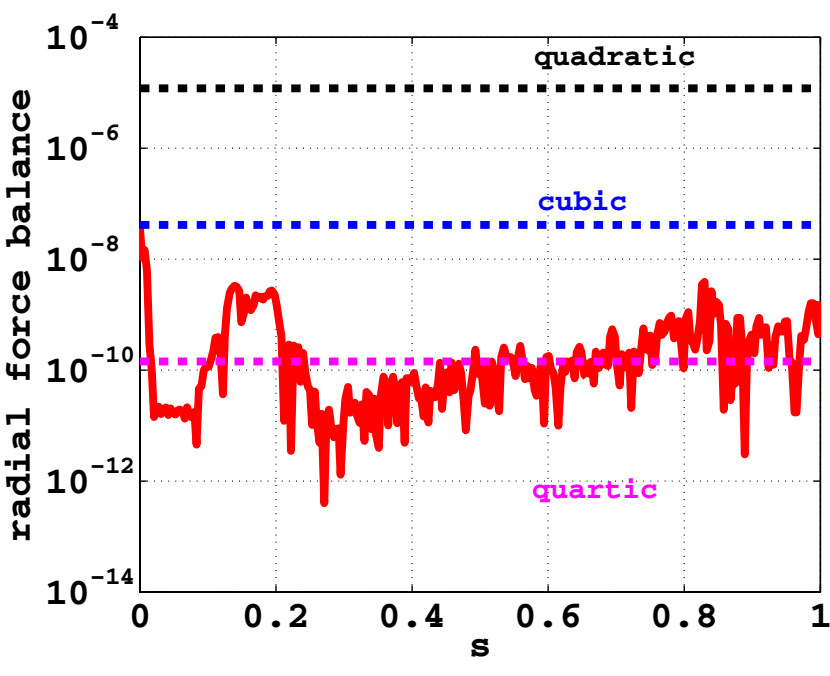

Figure 14. The radial force balance as a function of $s$. This shows that the convergence with radial mesh size is virtually quartic when the preconditioner is activated. Typically, quadratic convergence is expected. This compares very favourably with the radial force balance error reported in figure 2 of [6].

frequency $\Omega$ has a more direct impact on the equilibrium state. Hence, under condition of rigid toroidal flow $(\Omega \sim$ constant $)$, shear in the $U$ function can be manipulated with the radial profile of the temperature.

We have investigated LLM simulations of the MAST device including the effect of toroidal flow imposing fixed boundary conditions. We have specifically tailored the $\Omega(s)$ profile to be rigid within the confines of the 3D LLM deformation consistent with the experimental observations. We have succeeded in computing helical core equilibrium bifurcations with the toroidal flow model we have developed. We have limited the flow Mach number to $\mathcal{M}_{0}=0.3$ on axis as typically observed in MAST. The flow appears to have a negligible impact on the 3D magnetic structure at the subsonic levels relevant for LLM modelling. However, the rotation has an important effect on the pressure, displacing it radially outward so that it displays a complex combination of kink and centrifugal outward displacement. The rotational energy distribution is so strongly displaced away from the major axis that it appears virtually axisymmetric. Shear 
in the $U$-profile has only a small effect on the pressure distribution.

We have also exploited the equilibrium model to MAST simulations of externally applied up-down symmetric RMP under free boundary conditions. The main result obtained from these computations is the absence of rotational screening of the RMP deformations of the plasma with the model we have formulated. The underlying ideal Ohm's law innate to the background single fluid equilibrium state with nested magnetic flux surfaces we compute precludes the formation of island structures that can shield the external field perturbations. This corresponds to the reverse effect of plasma flow healing of vacuum magnetic islands in stellarators [26,27]. More general 3D codes that allow for general magnetic topology and incorporate a self-consistent plasma response to magnetic surface breaking must be considered to model this problem effectively. Equilibrium codes that allow field lines to break [28-30] would need to be extended.

\section{Acknowledgments}

WAC would like to thank Chris Hegna for very useful discussions and suggestions and to Ed Lazarus who encouraged this line of work. Some of the calculations were undertaken at the CSCS, Lugano, Switzerland under project ID:s493 and at IFERC, Rokkasho, Japan. This investigation was partially sponsored by the Fonds National Suisse de la Recherche Scientifique, by Euratom, by the US Department of Energy. The views and opinions expressed herein do not necessarily reflect those of the European Commission.

\section{References}

[1] Chapman I T et al 2010 Nucl. Fusion 50045007

[2] Graves J P et al 2013 Plasma Phys. Control. Fusion 55014005
[3] Menard J E et al 2005 Nucl. Fusion 45539

[4] Liu Y Q et al 2012 Plasma Phys. Control. Fusion 54124013

[5] Zehrfeld H P and Green B J et al 1972 Nucl. Fusion 12569

[6] Fitzgerald M, Appel L C and Hole M J 2013 Nucl. Fusion 53113040

[7] Clemente R A 1993 Nucl. Fusion 33963

[8] Hole M J and Dennis G 2009 Plasma Phys. Control. Fusion 51035014

[9] Guazzotto L, Betti R, Manickam J and Kaye S 2004 Phys. Plasmas 11604

[10] Hameiri E 1983 Phys. Fluids 26230

[11] Bondeson A and Iacono R 1990 Phys. Fluids B 11431

[12] Semenzato S, Gruber R and Zehrfeld H P 1984 Comput. Phys. Rep. 1389

[13] Park W et al 2003 Nucl. Fusion 43483

[14] Delgado-Aparicio Let al 2013 Phys. Rev. Lett. 110065006

[15] Sugiyama L E 2013 Phys. Plasmas 20032504

[16] Wang F, Fu G Y, Breslau J A, Tritz K and Liu J Y 2013 Phys. Plasmas 20072506

[17] Hirshman S P and Whitson J C 1983 Phys. Fluids 263553

[18] Hirshman S P, van Rij W I and Merkel P 1986 Comput. Phys. Commun. 43143

[19] Hirshman S P and Betancourt O 1991 J. Comput. Phys. 9699

[20] Maschke E and Perrin H 1980 Plasma Phys. 22579

[21] Cooper W A and Hirshman S P 1987 Plasma Phys. Control. Fusion 29933

[22] Cooper W A, Graves J P, Pochelon A, Sauter O and Villard L 2010 Phys. Rev. Lett. 105035003

[23] Cooper W A, Graves J P and Sauter O 2011 Plasma Phys. Control. Fusion 53024002

[24] Cooper W A, Graves J P and Sauter O 2011 Nucl. Fusion $\mathbf{5 1} 072002$

[25] Cooper W A et al 2013 Nucl. Fusion 53073021

[26] Hegna C C 2011 Nucl. Fusion 53024003

[27] Hegna C C 2012 Phys. Plasmas 19056101

[28] Suzuki Y et al 2006 Nucl. Fusion 46 L19

[29] Hirshman S P, Sanchez R, Lynch V E, D'Azevedo E F and Hill J C 2008 35th EPS Conf. on Plasma Physics (Hersonissos, Greece) vol 32D (ECA) P-2.058

[30] Hudson S R, Hole M J and Dewar R L 2007 Phys. Plasmas 14052505 